\title{
CRESCIMENTO DE Solanum lycocarpum St.-Hil. EM FUNÇÃO DA ADUBAÇÃO MINERAL E ORGÂNICA EM REJEITO DA MINERAÇÃO DE QUARTZITO
}

\author{
GROWTH OF Solanum lycocarpum St.-Hil. BY MINERAL AND ORGANIC FERTILIZATION ON \\ THE STERILE REJECT MINING QUARTZITE ${ }^{1}$
}

\author{
Cristiany Silva Amaral ${ }^{2}$ Enilson de Barros Silva ${ }^{3}$ Israel Marinho Pereira ${ }^{4}$ \\ Wander Gladson Amaral ${ }^{2}$ Vinicius de Morais Machado ${ }^{5}$
}

\begin{abstract}
RESUMO
Conduziu-se um experimento em casa de vegetação com o objetivo de avaliar o comportamento de Solanum lycocarpum sob a influência da adubação mineral e orgânica quando cultivada em rejeito da mineração de quartzito visando subsidiar ações voltadas para a recuperação de áreas de exploração de quartzito. Os tratamentos consistiram de cinco combinações de adubação orgânica (AO) e mineral (AM) e um tratamento adicional do rejeito sem $\mathrm{AO}$ e AM (Controle). As combinações foram: 0\%AO/100\%AM; 25\%AO/75\%AM; $50 \% \mathrm{AO} / 50 \% \mathrm{AM} ; 75 \% \mathrm{AO} / 25 \% \mathrm{AM} ; 100 \% \mathrm{AO} / 0 \% \mathrm{AM}$. As doses que corresponderam ao tratamento de $100 \%$ de AM e AO foram de $150 \mathrm{mg}$ de N, $140 \mathrm{mg}$ de $\mathrm{P}, 150 \mathrm{mg}$ de $\mathrm{K}$ e $5 \mathrm{~g}$ de esterco de curral por $\mathrm{dm}^{3} \mathrm{de}$ rejeito. A Solanum lycocarpum respondeu à adubação mineral e orgânica com as doses recomendadas de $36,0 \mathrm{mg} \mathrm{N}, 33,6 \mathrm{mg} \mathrm{P}$ e 36,0 mg K e 3,8 g de esterco de curral por $\mathrm{dm}^{3}$ de rejeito da mineração de quartzito. $\mathrm{O}$ acúmulo de nutrientes na espécie Solanum lycocarpum nas doses recomendadas foi na seguinte ordem na parte aérea: $\mathrm{N}>\mathrm{K}>\mathrm{Ca}>\mathrm{P}>\mathrm{S}>\mathrm{Mg}>\mathrm{Mn}>\mathrm{B}>\mathrm{Fe}>\mathrm{Cu}>\mathrm{Zn}$ com adubação mineral e orgânica para o máximo crescimento da espécie.
\end{abstract}

Palavras-chave: área degradada; lobeira; estado nutricional; teor adequado de nutriente.

\begin{abstract}
In order to evaluate the behavior of Solanum lycocarpum, grown in sterile tailings, from mining of quartzite under the influence of mineral and organic fertilization to support actions for the recovery of exploration areas of quartzite, it was conducted an experiment in a greenhouse in a completely randomized design with four replications The treatments consisted of five combinations of mineral (MF) and organic (OF) fertilizer and an additional treatment of the sterile reject without MF and OF (Witness). The combinations were: $0 \% \mathrm{OF} / 100 \% \mathrm{MF} ; 25 \% \mathrm{OF} / 75 \% \mathrm{MF} ; 50 \% \mathrm{OF} / 50 \% \mathrm{MF} ; 75 \% \mathrm{OF} / 25 \% \mathrm{MF} ; 100 \% \mathrm{OF} / 0 \% \mathrm{MF}$. Doses of $100 \%$ MF and OF were de $150 \mathrm{mg}$ de $\mathrm{N}, 140 \mathrm{mg}$ de $\mathrm{P}, 150 \mathrm{mg}$ de $\mathrm{K}$, e $5 \mathrm{~g}$ bovine manure per $\mathrm{dm}^{3}$ of sterile. The Solanum lycocarpum responded to mineral fertilization with the recommended doses of $36 \mathrm{mg} \mathrm{N}, 33,6 \mathrm{mg}$ $\mathrm{P}$ e $36 \mathrm{mg} \mathrm{K}$ e 3,8 $\mathrm{g}$ bovine manure per $\mathrm{dm}^{3}$ of sterile tailings from mining of quartzite. The accumulation of nutrients in the species Solanum lycocarpum at recommended doses in the following order was on the

1 Parte da Dissertação do primeiro autor apresentada no Programa de Pós-Graduação em Ciência Florestal da Universidade Federal dos Vales do Jequitinhonha e Mucuri (UFVJM), Diamantina, MG.

2 Engenheiro Florestal, Doutorando em Ciência Florestal, Universidade Federal dos Vales do Jequitinhonha e Mucuri, Campus JK, Rodovia MGT 367, 5000, CEP 39100-000, Diamantina (MG), Brasil. Bolsista CAPES. cristianyamaral@hotmail.com / wander.amaral@yahoo.com.br

3 Engenheiro Agrônomo, Dr., Professor Associado do Departamento de Agronomia, Universidade Federal dos Vales do Jequitinhonha e Mucuri, Campus JK, Rodovia MGT 367, 5000, CEP 39100-000, Diamantina (MG), Brasil. ebsilva@ufvjm.edu.br

4 Engenheiro Florestal, Dr., Professor Adjunto do Departamento de Engenharia Florestal, Universidade Federal dos Vales do Jequitinhonha e Mucuri, Campus JK, Rodovia MGT 367, 5000, CEP 39100-000, Diamantina (MG), Brasil. imarinhopereira@gmail.com

5 Engenheiro Florestal, Doutorando em Ciência Florestal, UNB-Brasília. Campus Universitário Darcy Ribeiro, 4357, Asa Norte, CEP 39100-000, Brasília (DF). Bolsista do CAPES. viniciusfloresta@hotmail.com.
\end{abstract}

Recebido para publicação em 18/07/2012 e aceito em 23/01/2018

Ci. Fl., v. 28, n. 4, out. - dez., 2018 
shoot: $\mathrm{N}>\mathrm{K}>\mathrm{Ca}>\mathrm{P}>\mathrm{S}>\mathrm{Mg}>\mathrm{Mn}>\mathrm{B}>\mathrm{Fe}>\mathrm{Cu}>\mathrm{Zn}$ with fertilization mineral and organic for maximum growth of the of species.

Keywords: degraded area; 'lobeira'; nutritional status; nutrients accumulation;

\section{INTRODUÇÃO}

O Município de Diamantina situa-se na Cadeia do Espinhaço que é reconhecida internacionalmente em função do elevado grau de endemismo concentrado em seu território (ALVES et al., 2014).

Com o declínio da mineração de diamante, a exploração de maciços de quartzito se torna frequente; o que provoca degradação do solo e a extinção de espécies endêmicas em razão da forte ação antrópica (CARMO, 2012).

A atividade de mineração promove alto grau de impacto, pois as áreas deixadas com rejeito, subsolo ou cascalho exposto permanecem destituídas de vegetação mesmo tendo sido abandonadas por décadas (DURÃES et al., 2017), com baixa resiliência (CULLEN; WHEATER; DUNLEAVY, 1998). O reduzido restabelecimento natural das espécies deve-se ao fato de haver a remoção da serapilheira, que contém sementes de plantas herbáceas, arbustivas e arbóreas que formam o banco de sementes e de plântulas que são, partes integrantes da população vegetal (JESUS et al., 2016).

Dentre as espécies que podem ser indicadas para compor os plantios nessas áreas degradadas destaca-se a espécie Solanum lycocarpum (SILVA et al., 2016), conhecida popularmente como lobeira ou fruta-de-lobo (OLIVEIRA JUNIOR et al., 2003) pertence à família Solanaceae, é uma espécie arbórea típica do cerrado brasileiro (RIZZINI, 1963; ROESLER et al., 2007), indicada para recuperação de áreas degradadas devido a sua rápida germinação, alta taxa de emergência de plântulas (BEZERRA et al., 2016), crescimento rápido e facilidade da sucessão ecológica nos estágios iniciais (OLIVEIRA, 2006). A modificação do ambiente pela lobeira pode ser avaliada dentre outros fatores, em termos de estrutura do solo, conteúdo de nutrientes e matéria orgânica, pois são variáveis que podem impedir ou facilitar o estabelecimento de espécies (HOLL, 2000).

Dessa maneira, a lobeira apresenta atributos que justificam seu uso em plantios com fins para a restauração ambiental, no entanto, o pouco conhecimento do comportamento dessa espécie, principalmente em relação às suas exigências nutricionais em áreas degradadas pela mineração de quartzito torna sua utilização comprometida em programas de restauração.

O objetivo do trabalho foi de avaliar o comportamento de Solanum lycocarpum sob a influência da adubação mineral e orgânica quando cultivada em rejeito da mineração de quartzito para apoiar ações voltadas para a recuperação dessas áreas de exploração.

\section{MATERIAL E MÉTODOS}

O experimento foi conduzido de janeiro de 2011 a abril de 2011, em condições de casa de vegetação no Campus JK da Universidade Federal dos Vales do Jequitinhonha e Mucuri (UFVJM), em Diamantina-MG, Brasil (18 $12^{\circ}$ 'S; $43^{\circ} 34^{\prime} \mathrm{W}$ e altitude de $\left.1.370 \mathrm{~m}\right)$.

Para instalação do experimento utilizou-se o rejeito da mineração de quartzito oriundo das áreas de exploração localizada no município de Diamantina-MG, do qual foi retirada uma amostra para análise química (SILVA, 2009) e de textura (EMBRAPA, 1997) do substrato.

A amostra do substrato foi seca ao ar por 48 horas, e passada em peneira de 2,0 $\mathrm{mm}$ de abertura. Os resultados da análise química foram ajustados para o todo da constituição granulométrica dos rejeitos de quartzito (Tabela 1). 
TABELA 1: Análise química e de textura do rejeito da mineração de quartzito.

TABLE 1: Chemical analysis and texture of reject from mining of quartzite.

\begin{tabular}{|c|c|c|c|c|c|c|c|c|c|c|c|c|}
\hline \multirow[t]{2}{*}{$\mathrm{pH}_{\text {àgua }}$} & $\mathrm{P}$ & $\mathrm{K}$ & $\mathrm{Ca}$ & $\mathrm{Mg}$ & $\mathrm{Al}$ & $\mathrm{T}$ & $\mathrm{m}$ & V & MO & $\begin{array}{c}\text { Cascalho/ } \\
\text { Calhaus }\end{array}$ & Silte & Argila \\
\hline & \multicolumn{2}{|c|}{$-m g d m^{-3}-$} & \multicolumn{4}{|c|}{----- $\mathrm{cmol}_{\mathrm{c}} \mathrm{dm}^{-3}$------ } & \multicolumn{2}{|c|}{---- \%----- } & \multicolumn{4}{|c|}{----------------- g kg $^{-1}$--------------- } \\
\hline 5,0 & 1,2 & 5,3 & 0,5 & 0,2 & 0,2 & 1,7 & 24 & 41 & 7,1 & 340,4 & 99 & 6,6 \\
\hline
\end{tabular}

Em que: $\mathrm{pH}_{\text {água }}$ - relação solo-água 1:2,5; P e K - Extrator Mehlich-1; Ca, Mg e Al - Extrator KCl 1 mol L-1; T - Capacidade de troca de cátions a pH 7,0; m - Saturação de alumínio; V - Saturação por bases; MO - Matéria orgânica determinado através da multiplicação do resultado do carbono orgânico pelo método Walkey-Black por 1,724; Cascalho/ calhaus - Tamisação em peneira de 2,0 mm; Areia, silte e argila - Método da pipeta.

O delineamento experimental utilizado foi inteiramente casualizado com cinco combinações de adubação orgânica (AO) e mineral (AM) e um tratamento adicional (Controle), com quatro repetições. As combinações são apresentadas na Tabela 2.

TABELA 2: Descrição dos tratamentos aplicados.

TABLE 2: Description of treatments applied.

\begin{tabular}{cccccc}
\hline $\begin{array}{c}\text { Adubação } \\
\text { mineral (AM) }\end{array}$ & $\begin{array}{c}\text { Adubação orgânica } \\
(\mathrm{AO})\end{array}$ & $\mathrm{N}$ & $\mathrm{P}$ & $\mathrm{K}$ & Esterco curral \\
& \multicolumn{2}{c}{$\ldots \ldots \ldots \ldots . . \mathrm{mg} \mathrm{dm}{ }^{-3} \ldots \ldots \ldots \ldots$} & $\ldots \ldots . . \mathrm{g} \mathrm{dm}^{-3} \ldots \ldots \ldots$ \\
\hline $100 \%$ & $0 \%$ & 150 & 140 & 150 & 0 \\
$75 \%$ & $25 \%$ & 112,5 & 105 & 112,5 & 1,25 \\
$50 \%$ & $50 \%$ & 75 & 70 & 75 & 2,5 \\
$25 \%$ & $75 \%$ & 37,5 & 35 & 37,5 & 3,75 \\
$0 \%$ & $100 \%$ & 0 & 0 & 0 & 5 \\
\hline & Controle & 0 & 0 & 0 & 0 \\
\hline
\end{tabular}

As doses que corresponderam a $150 \mathrm{mg}$ de $\mathrm{N}$ por $\mathrm{dm}^{3}$ de rejeito, e $150 \mathrm{mg}$ de $\mathrm{K}$ por $\mathrm{dm}^{3}$ de rejeito foram recomendadas segundo trabalho realizado por Venturin et al. (2005), no qual avaliaram os aspectos nutricionais e os efeitos da omissão de nutrientes no desenvolvimento de plântulas de candeia (Eremanthus erythropappus (DC.) McLeish). A dose que correspondia ao trabalho de Venturin et al. (2005), a $200 \mathrm{mg}$ de $\mathrm{P}$ por $\mathrm{dm}^{3}$ de Latossolo Vermelho-Amarelo, sofreu uma redução para $140 \mathrm{mg}$ de $\mathrm{P}$ por $\mathrm{dm}^{3}$ de rejeito, por se tratar de substrato de textura muito arenosa (MACHADO et al., 2011). N, P e K foram aplicados na forma de sulfato de amônio, superfosfato simples e cloreto de potássio, respectivamente. A dose de $5 \mathrm{~g}$ de esterco de curral por $\mathrm{dm}^{3}$ de rejeito foi recomendada segundo a Comissão de Fertilidade do Solo do Estado de Minas Gerais (1999). As análises químicas do esterco de curral $(\mathrm{pH}=6,8 ; \mathrm{N}=6,0 ; \mathrm{P}=1,5 ; \mathrm{K}=1,3 ; \mathrm{Ca}=$ 0,$3 ; \mathrm{Mg}=0,4 ; \mathrm{S}=0,8 ; \mathrm{B}=0,18 ; \mathrm{Cu}=0,008 ; \mathrm{Fe}=27,2 ; \mathrm{Mn}=0,13$ e $\mathrm{Zn}=0,065 \mathrm{~g} \mathrm{~kg}$-1; C-orgânico $=178 \mathrm{~g}$ $\mathrm{kg}-1$; e densidade $=0,69 \mathrm{~kg} \mathrm{dm}-3$ ) foram realizadas segundo metodologia descrita por Melo e Silva (2008).

A parcela experimental foi composta por vasos de plástico de $5 \mathrm{dm}^{3}$ nos quais foram colocados 3 $\mathrm{dm}^{3}$ de rejeito de quartzito seco, que obteve densidade uniforme de $0,93 \mathrm{~g} \mathrm{~cm}^{-3}$. Os vasos tiveram o fundo fechado para evitar perda de nutrientes e de água.

$\mathrm{Na}$ instalação do experimento foram aplicados $0,119 \mathrm{~g}$ de calcário dolomítico por $\mathrm{dm}^{3}$ de rejeito em todos os tratamentos com o tempo de incubação de 30 dias, para fornecimento de $\mathrm{Ca}$ e $\mathrm{Mg}$ pelo método da saturação por bases (ALVAREZ; BARROS, 1999) para elevar para $55 \%$. Na mesma época foi realizada a adubação fosfatada e a adubação orgânica conforme descrito nos tratamentos. $\mathrm{O}$ material de rejeito foi 
incubado por mais 30 dias, sendo a umidade do material mantida durante todo o experimento em $60 \%$ do volume total de poros (VTP), aferida diariamente através de pesagem, completando-se o peso com água deionizada.

Uma semana antes do plantio das sementes foi feita a adubação básica de B (ácido bórico) e Zn (sulfato de zinco) na dose de 0,0005 g de B e 0,001 g de Zn por dm³ de rejeito (CANTARUTTI et al., 1999).

As sementes de lobeira foram coletadas em 15 árvores selecionadas no campo e colocadas 15 sementes a germinar diretamente nos vasos contendo o rejeito de quartzito, 30 dias após a germinação das sementes foi feito o desbaste deixando-se apenas uma planta por vaso. As adubações potássica e nitrogenada em cobertura iniciaram 15 dias após o desbaste e foram parceladas em quatro vezes, sendo as aplicações realizadas em intervalos de 15 dias.

Após um período experimental de 120 dias foram avaliadas as seguintes variáveis: altura das mudas, medida do colo até a gema apical obtida entre a superfície do substrato e a inserção do último par de folhas do ápice, com régua graduada de precisão de $1,0 \mathrm{~mm}$; diâmetro do caule medido na altura do colo obtido com auxílio de um paquímetro digital com precisão de $0,01 \mathrm{~mm}$.

Depois de medidos os diâmetros e as alturas, as mudas foram cortadas rente ao substrato. O sistema radicular foi separado do substrato. Raízes e parte aérea, após coletadas foram separadas por tratamentos e repetições, lavadas em água destilada, acondicionadas em sacos de papel e secas em estufa com circulação forçada de ar, à temperatura de $65^{\circ} \mathrm{C}$ por 72 horas até atingir peso constante para determinação da massa seca. Após a secagem, o material foi pesado em balança analítica com precisão de $0,0001 \mathrm{~g}$, obtendo o peso de massa seca da parte aérea e das raízes.

Para avaliação do estado nutricional de Solanum lycocarpum sob AO e AM quando cultivada em rejeito da mineração de quartzito, após moagem do material, foram determinados os teores de macro e micronutrientes na massa seca da parte aérea segundo metodologia descrita por Malavolta, Vitti e Oliveira (1997). Para o cálculo dos acúmulos de nutrientes da parte aérea da lobeira multiplicou-se o teor foliar do nutriente na massa seca da parte aérea e, dessa forma, obteve-se o acúmulo do nutriente na parte aérea da espécie.

Os dados obtidos foram submetidos à análise de variância. Para se obter a porcentagem de AM e AO para melhor crescimento das mudas de lobeira utilizou-se de um procedimento multivariado através do processo da variável canônica (MORRISON, 1967). Com a variável canônica de maior autovalor obtiveram-se os escores a partir do vetor observação de cada unidade experimental das variáveis de crescimento das mudas de lobeira estudadas, reduzindo-o para único valor. Tais escores foram submetidos à análise de variância univariada e as porcentagens de AM foram submetidas ao estudo de regressão polinomial e a média do controle foi comparada com os demais tratamentos pelo teste de $\mathrm{F}$.

$\mathrm{O}$ teor foliar adequado e acumulado de nutrientes na parte aérea da lobeira foi estimado substituindo a porcentagem de AM para o máximo crescimento da espécie nas equações que relacionam a porcentagem de AM com essas variáveis. Foi utilizado o programa SAS for Windows pelo procedimento PROC GLM e REG para os procedimentos estatísticos.

\section{RESULTADOS E DISCUSSÃO}

As características de crescimento das mudas de lobeira apresentaram influência da adubação mineral (AM) e orgânica (AO) aplicada no rejeito da mineração de quartzito (Tabela 3). O tratamento controle (sem $\mathrm{AM} \mathrm{e} \mathrm{AO}$ ) é inferior às demais combinação de $\mathrm{AM}$ e $\mathrm{AO}$ quanto às variáveis de crescimento da espécie (Tabela 3 ). 
TABELA 3: Diâmetro do caule, altura de mudas, massa seca da parte aérea (MSPA) e das raízes (MSR) e relação de MSR e MSPA de mudas de lobeira aos 120 dias em função da porcentagem de adubação mineral (AM) e orgânica (AO) aplicada em rejeito da mineração de quartzito

TABLE 3: Stem diameter, plant height, shoot dry mass (MSPA) and roots (MSR) and relationship MSR e MSPA of seedlings of lobeira at 120 days, depending on the percentage of the mineral (AM) and organic fertilizers (AO) applied to reject the mining of quartzite

\begin{tabular}{|c|c|c|c|c|c|c|}
\hline AM & $\mathrm{AO}$ & $\begin{array}{c}\text { Diâmetro } \\
(\mathrm{mm})\end{array}$ & $\begin{array}{l}\text { Altura } \\
(\mathrm{cm})\end{array}$ & $\begin{array}{c}\text { MSPA } \\
(\mathrm{g})\end{array}$ & $\begin{array}{c}\text { MSR } \\
(\mathrm{g})\end{array}$ & $\begin{array}{c}\text { Relação } \\
\text { MSR/MSPA }\end{array}$ \\
\hline 100 & 0 & 1,97 & 4,25 & 0,26 & 0,04 & 0,19 \\
\hline 75 & 25 & 2,17 & 6,63 & 0,31 & 0,07 & 0,2 \\
\hline 50 & 50 & 2,89 & 8,75 & 0,5 & 0,25 & 0,49 \\
\hline 25 & 75 & 5,91 & 20,83 & 1,74 & 0,85 & 0,5 \\
\hline 0 & 100 & 5,08 & 17,38 & 1,19 & 0,38 & 0,3 \\
\hline \multicolumn{2}{|c|}{ Controle $^{(1)}$} & 3,05 & 9,45 & 0,34 & 0,12 & 0,33 \\
\hline \multicolumn{2}{|c|}{ Média } & 3,51 & 11,21 & $0,72 *$ & $0,28 *$ & 0,34 \\
\hline \multicolumn{2}{|c|}{$\mathrm{CV}(\%)$} & 21,88 & 23,72 & 32,9 & 37,99 & 24,59 \\
\hline
\end{tabular}

Em que: *Diferença do controle das porcentagens de adubação mineral e orgânica pelo teste $\mathrm{F}$ a 5\%.

Pelos escores de crescimento das mudas de lobeira da variável canônica $(\mathrm{VC}=-2,5418 *$ Diâmetro - 0,0883*Altura - 10,0191*MSPA + 29,0957*MSR + 1,2779*Relação MSR/MSPA com autovalor $=96$ $\%)$ verificou-se que o máximo crescimento foi atingido com a porcentagem de $24 \%$ de $\mathrm{AM} \mathrm{e} 76 \%$ de AO (Figura 1), que correspondeu às doses de 36,0 mg N, 33,6 mg P e 36,0 mg K e 3,8 g de esterco de curral por $\mathrm{dm}^{3}$ de rejeito da mineração de quartzito.

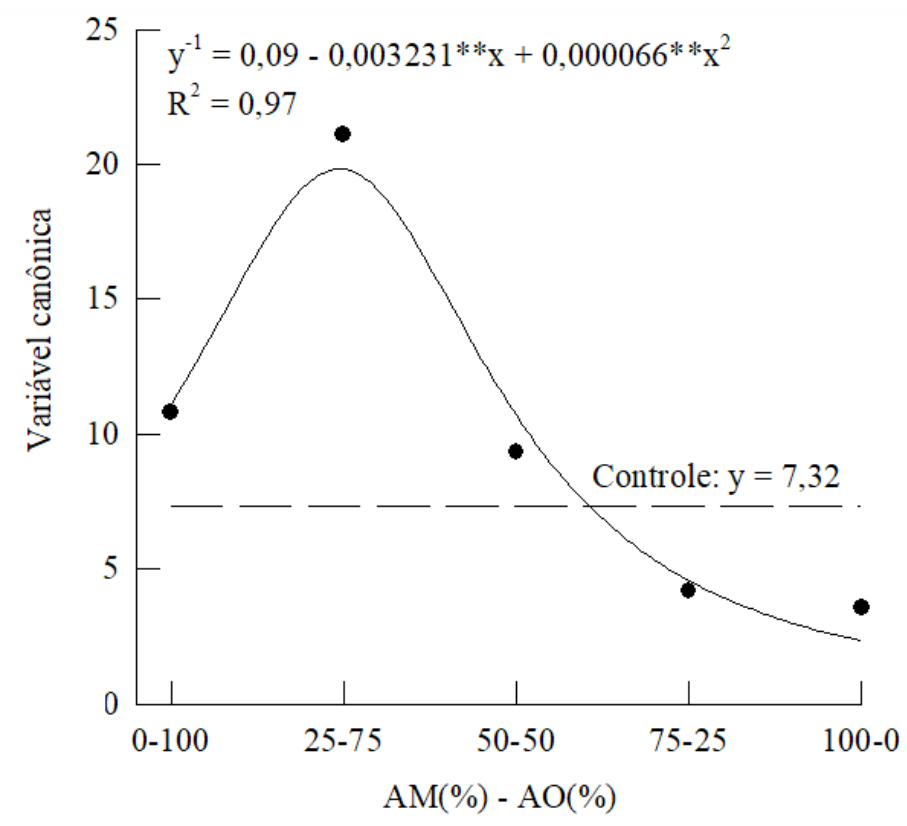

FIGURA 1: Escore da variável canônica da análise multivariada em função da porcentagem de adubação mineral (AM) e orgânica (AO) aplicada em rejeito da mineração de quartzito no crescimento de mudas de lobeira $(* *$ significativo a $1 \%$ pelo teste de $\mathrm{t}$ ).

FIGURE 1: Score of the canonical variable of the multivariate analysis according to the percentage of mineral fertilizer (AM) and organic (AO) applied to reject from mining of quartzite in the growth of seedlings of lobeira $(* *$ significant at $1 \%$ by test $t)$. 
As combinações não adequadas de AM e AO mostraram-se limitantes, afetando em geral o crescimento das mudas de lobeira em altura, diâmetro e produção de massa seca da parte aérea e das raízes (Tabela 3). O maior crescimento da espécie, observado com a combinação de $24 \% \mathrm{AM} \mathrm{e} 76 \% \mathrm{AO}$ em relação aos demais tratamentos e ao controle, se deve ao efeito positivo da matéria orgânica combinada adequadamente com a adubação mineral. Este efeito no crescimento pode ser relacionado com as modificações nas propriedades físicas do substrato, que por sua vez, influencia na capacidade de infiltração, retenção de água, drenagem, aeração, temperatura e penetração do sistema radicular (KANG, 1991; VARANINE et al., 1993).

Em termos de alteração na partição de matéria seca, entre raízes e parte aérea (relação R/PA), diante das mudanças de disponibilidade dos nutrientes em função das combinações de adubação, observou-se que a lobeira teve maior relação na combinação de $24 \%$ AM e 76 \% AO apresentando relação de 0,50 (Tabela 3), valor este dentro da faixa de relação ideal para produção de mudas a serem levadas ao campo entre 0,45 a 0,50 (DANIEL; VITORINO; ALOVISI, 1997). A relação R/PA em mudas de Acacia mangiun foi de 0,45 pela aplicação de $260 \mathrm{mg} \mathrm{dm}^{-3}$ de P (DANIE; VITORINO; ALOVISI, 1997) enquanto que em mudas de jatobá foi de 0,6 no tratamento completo e de 0,51 quando da omissão de cálcio (DUBOC et al., 1996) sendo que as doses de $\mathrm{N}, \mathrm{P}$ e $\mathrm{K}$ aplicadas no tratamento completo consistiram de $150 \mathrm{mg} \mathrm{kg}^{-1} ; 120 \mathrm{mg} \mathrm{kg}^{-1}$; $75 \mathrm{mg} \mathrm{kg}^{-1}$, respectivamente.

Resposta positiva à adubação mineral e orgânica foram encontradas em Erva-mate (Ilex paraguariensis St.Hil.) cultivada em Latossolo Vermelho com aplicação de $80,5 \mathrm{~g}$ de N, 25g de $\mathrm{P}_{2} \mathrm{O}_{5}, 75 \mathrm{~g}$ de $\mathrm{K}_{2} \mathrm{O}$ e 4,5 kg de cama de aviário por planta (PANDOLFO et al., 2003). Em espécies nativas conhecidas como aroeira-pimenteira (Schinus terebinthifolia Raddi), cabreúva-vermelha (Myroxylon peruiferum L.f.), pau-de-viola (Cytarexyllum myrianthum Cham) e unha-de-vaca (Bauhinia forficata Link), adubadas com $20 \mathrm{~g}$ $\mathrm{dm}^{-3}$ de logo de esgoto (adubação orgânica) e com 0,42 g de KCL e 2,5 g de calcário (PAIVA et al., 2009). Em Stryphnodendron polyphyllum (barbatimão) com aplicação de N (12 g/cova equivalente a $375 \mathrm{~kg} \mathrm{ha}^{-1}$ de sulfato de amônio) e P (123,7 g/cova, equivalente a $3.865 \mathrm{~kg} \mathrm{ha}^{-1}$ de surperfosfato simples), associada aos resíduos orgânicos (954 g/cova, equivalente a $30 \mathrm{t} \mathrm{ha}^{-1}$ de material seco) (CALGARO et al., 2008). E em três espécies florestais (Cedrela odorata, Schinnus terebinthifolius e Acacia holosericea) com aplicação de adubo orgânico nas doses recomendadas de 1 litro/cova de esterco de galinha e de esterco bovino e com aplicação de 56 g/cova de NPK-20-05-20 (SOUZA et al., 2006).

Os teores de nutrientes foram influenciados pela AM e AO que diferiram do controle, exceto o teor de K e B na parte aérea da espécie (Tabela 4). A avaliação do estado nutricional de Solanum lycocarpum permitiu obter os teores adequados de macro e micronutrientes (Tabela 4) para a espécie em área degradada pela extração de quartzito em função da $\mathrm{AM}$ e $\mathrm{AO}$ que proporcionaram o máximo crescimento.

$\mathrm{O}$ teor foliar adequado de $\mathrm{N}$ na lobeira cultivada em rejeito de quartzito com $24 \%$ de AM e $76 \%$ AO no qual proporcionou o maior crescimento das plantas de lobeira aos 120 dias (Tabela 4) está dentro da faixa adequada para essências florestais preconizada por Malavolta, Vitti e Oliveira (1997) que varia de 12 a $35 \mathrm{~g} \mathrm{~kg}^{-1}$ confirmando a importância recíproca da $\mathrm{AM}$ e $\mathrm{AO}$ quanto à disponibilidade de $\mathrm{N}$ para atender à demanda nutricional da espécie.

No estádio juvenil, a demanda de N é muito elevada (GONÇALVES et al., 2000) e a maior absorção e assimilação de $\mathrm{N}$ é aceita como uma das principais causas da resposta em crescimento (VAZ; GONÇALVES, 2002). Ao comparar o teor de $\mathrm{N}$ entre o controle e o $24 \%$ AM e $76 \%$ AO, observa-se que o teor no controle (Tabela 4) foi inferior, no entanto, está dentro da faixa adequada (MALAVOLTA; VITTI; OLIVEIRA, 1997), esta resposta deve-se possivelmente ao efeito da concentração (MARQUES et al., 2004) devido ao menor crescimento da planta no controle (Tabela 3).

Estes resultados são semelhantes aos obtidos por Wallau et al. (2008) em experimento para determinar as concentrações de nutrientes na parte aérea de plantas de cedro cultivadas em solução nutritiva completa, e por Duboc e Guerrini (2008) quando avaliaram a concentração foliar de N na espécie conhecida como angico sob adubação mineral $\mathrm{N}$ e $\mathrm{P}$ em condições não degradadas.

A concentração do P na parte aérea de lobeira na combinação de $26 \%$ AM e $74 \%$ AO (Tabela 4) encontra-se acima da faixa adequada de 1,0 a 2,3 $\mathrm{g} \mathrm{kg}^{-1}$ (MALAVOLTA; VITTI; OLIVEIRA, 1997) para essências florestais. Esse comportamento pode ser explicado pela textura arenosa do substrato e pela presença de matéria orgânica via aplicação de esterco bovino. Essa disponibilidade pode ser influenciada pela textura do solo, sendo que a adsorção de $\mathrm{P}$ em solos arenosos é menor que em solos com maior teor de argila 
(FALCÃO; SILVA, 2004) e que a adsorção de fosfato é menor na presença de matéria orgânica (BHATTI; COMEFORD; JOHNSTON, 1998), além da matéria orgânica ser fonte de P para as plantas (VIEIRA et al., 1993; RHEINHEIMER; ANGHINONI; CONTE, 2000; CASSOL; GIANELLO; COSTA, 2012).

Já no controle, como era esperado, o teor de P na parte aérea está deficiente (abaixo da faixa adequada), uma vez que a disponibilidade de P no solo é um fator que interfere diretamente na sua absorção pelas plantas (MACHADO et al., 2011). Isso demonstra que a quantidade aplicada de P no melhor tratamento para obter o máximo crescimento foi suficiente para atender à demanda nutricional e que a adubação fosfatada é responsiva no crescimento inicial e na produção de lobeira.

TABELA 4: Equações de regressão ajustadas para os teores de macro e micronutrientes na parte aérea de lobeira em função da porcentagem de adubação mineral aplicada em rejeito da mineração de quartzito, teor de nutrientes correspondente a $24 \%$ de adubação mineral (AM) e no controle, em um período de 120 dias.

TABLE 4: Regression equations adjusted to the levels of macro and micronutrients in the shoot of lobeira according to the percentage of mineral fertilizer applied to tailings from mining of quartzite, nutrient concentration corresponding to $24 \%$ of mineral fertilizer (AM) and in control, in a period 120 days.

\begin{tabular}{|c|c|c|c|c|}
\hline Nutriente $^{(1)}$ & Equação de Regressão & $\mathrm{R}^{2}$ & $\mathrm{AM}^{(2)}$ & Controle $^{(3)}$ \\
\hline $\mathrm{N}$ & $\hat{\mathrm{y}}=12,24+0,4915^{* *} \mathrm{x}-0,0043^{* *} \mathrm{x}^{2}$ & 0,99 & 21,75 & $18,52 *$ \\
\hline $\mathrm{P}$ & $\hat{y}=2,48+0,1118^{* *} x-0,0009^{* *} x^{2}$ & 0,88 & 4,64 & $0,38^{*}$ \\
\hline $\mathrm{K}$ & $\hat{\mathrm{y}}=\overline{\mathrm{y}}=16,91$ & - & $16,91^{(4)}$ & $10,11^{*}$ \\
\hline $\mathrm{Ca}$ & $\hat{y}=3,96+0,1175^{* * x}$ & 0,89 & 6,78 & $13,79 *$ \\
\hline $\mathrm{Mg}$ & $\hat{\mathrm{y}}=0,37+0,0174 * * x$ & 0,95 & 0,79 & $0,80^{*}$ \\
\hline $\mathrm{S}$ & $\hat{y}=3,57-0,0304 * * x$ & 0,95 & 2,84 & $0,70 *$ \\
\hline $\mathrm{B}$ & $\hat{\mathrm{y}}=\overline{\mathrm{y}}=68,01$ & - & $68,01^{(4)}$ & 69,63 \\
\hline $\mathrm{Cu}$ & $\hat{y}=13,69+0,2086^{* *} x$ & 0,98 & 18,69 & $29,12 *$ \\
\hline $\mathrm{Fe}$ & $\hat{y}=2,69+8,4955^{* *} x-0,0529^{* *} x^{2}$ & 0,85 & 176,1 & $136,81^{*}$ \\
\hline $\mathrm{Mn}$ & $\hat{y}=385,16-6,0282 * * x+0,0384 * * x^{2}$ & 0,86 & 262,67 & $176,45^{*}$ \\
\hline $\mathrm{Zn}$ & $\hat{y}=11,41+0,2976^{* *} x-0,0039 * * x^{2}$ & 0,8 & 16,31 & $5,06^{*}$ \\
\hline
\end{tabular}

Em que: **significativo a $1 \%$ pelo teste de t; (1)Macronutrientes em g kg-1 e micronutrientes em mg kg-1; (2) Teor nutriente na aplicação de $24 \%$ AM e 76 \% adubação orgânica (AO); (3) * Diferença do controle dos demais tratamentos pelo teste F a 5\%; (4) Teor médio das combinações de AM e AO.

O P é um nutriente considerado determinante no crescimento das plantas, uma vez que tem considerável importância no metabolismo do carbono, bem como na formação de açúcares fosfatados (GRANT et al., 2001). Ao se comparar o teor de P obtidos no melhor tratamento (24\% AM e $76 \%$ AO) com trabalhos de outros autores observa-se que o teor de $\mathrm{P}$ na melhor combinação de AM e AO está próximo aos encontrados por Marques et al. (2004) para Schizolobium amazonicum no qual obtiveram teor de $4,3 \mathrm{~g} \mathrm{~kg}^{-1} \mathrm{e} \mathrm{por}^{-}$ Sorreano et al. (2011) avaliando exigência nutricional na espécie sangra-d'água (Croton urucurana) na qual obtiveram teor de $\mathrm{P}$ de $5,5 \mathrm{~g} \mathrm{~kg}^{-1}$.

Verifica-se que o teor de $\mathrm{P}\left(0,38 \mathrm{~g} \mathrm{~kg}^{-1}\right)$ no tratamento controle foi semelhante ao observado por 
Duboc et al. (1996) estudando a nutrição do jatobá (Hymenaea courbaril L. var. stilbocarpa (Hayne) Lee et Lang.) no qual obtiveram $0,4 \mathrm{~g} \mathrm{~kg}^{-1}$, indicando uma elevada capacidade de extração pelas mudas de lobeira, mesmo em condições de pequena disponibilidade no substrato.

Com $24 \%$ AM e $76 \%$ AO, verifica-se que o teor foliar de K para a espécie está acima da faixa adequada de 10 a15 $\mathrm{g} \mathrm{kg}^{-1}$ (MALAVOLTA; VITTI; OLIVEIRA, 1997) para essências florestais. Verifica-se na parte aérea do tratamento controle que o teor de $\mathrm{K}$ foi menor quando comparado ao tratamento com maior crescimento (Tabela 4), no entanto, o teor no controle também se encontra dentro da faixa adequada, possivelmente devido ao efeito da concentração.

Resultados semelhantes ao tratamento com maior crescimento foram observados em Marques et al. (2004) em plantas de paricá com aplicação de solução nutritiva completa. Enquanto Duboc et al. (1996) obtiveram teores de $\mathrm{K}$ inferiores com plantas de jatobá com aplicação total de $75 \mathrm{mg} \mathrm{dm}^{-3}$ de solo. Sorreano et al. (2011) obtiveram teores de K superiores aos obtidos neste trabalho quando avaliaram mudas de Croton urucurana na adubação completa em solução nutritiva.

$\mathrm{Na}$ massa seca da parte aérea de lobeira cultivada em rejeito de quartzito com $24 \%$ de AM e $76 \%$ $\mathrm{AO}$, o teor foliar de Ca está dentro da faixa adequada enquanto o teor de $\mathrm{Mg}$ (Tabela 4) se encontra abaixo da faixa adequada, preconizada por Malavolta, Vitti e Oliveira (1997) para essências florestais de 3 a1 $2 \mathrm{~g}$ $\mathrm{kg}^{-1}$ de Ca e de 1,5 a 5,0 $\mathrm{g} \mathrm{kg}^{-1}$ de $\mathrm{Mg}$. A adubação potássica promoveu altas concentrações de $\mathrm{K}$ na solução do rejeito que reduziu a absorção de $\mathrm{Mg}$ por inibição competitiva pelos mesmos sítios de absorção (MARSCHNER, 1995).

O teor foliar de Ca encontrado com aplicação de $24 \%$ de AM e 76 \% AO está abaixo ao encontrados por Viégas et al. (2002) para Annona muricata $\left(14,1 \mathrm{~g} \mathrm{~kg}^{-1}\right)$ e por Sorreano et al. (2011) para Croton urucurana $\left(15,8 \mathrm{~g} \mathrm{~kg}^{-1}\right)$. Desta forma, fazem-se necessários estudos de recomendação de Ca e Mg para ambientes degradados pela mineração de quartzito.

Quanto ao macronutriente S, o teor do controle diferiu das combinações de AM e AO (Tabela 4). Enquanto que com $24 \% \mathrm{AM}$ e $76 \% \mathrm{AO}$, o teor de $\mathrm{S}$ na parte área da lobeira (Tabela 4) se encontra dentro da faixa adequada, já no controle, o teor encontra-se abaixo da faixa adequada de 1,4 a1,6 $\mathrm{g} \mathrm{kg}^{-1}$ (MALAVOLTA; VITTI; OLIVEIRA, 1997) para espécies florestais. O maior teor de S na melhor combinação de $\mathrm{AM}$ e $\mathrm{AO}$ pode ser explicado pela própria capacidade do substrato em disponibilizar S, pelo uso do sulfato de amônio como fonte de $\mathrm{N}$, que correspondeu à aplicação $0,18 \mathrm{~g} \mathrm{dm}^{-3}$ do rejeito; e ainda, pela quantidade de matéria orgânica adicionada nesse tratamento. O teor de S com $24 \%$ AM e 76 \% AO foi superior ao obtido por Duboc et al. (1996) de $0,65 \mathrm{~g} \mathrm{~kg}^{-1} \mathrm{de} \mathrm{S}$ com aplicação de $30 \mathrm{mg} \mathrm{dm}^{-3}$ de $\mathrm{S}$ no solo, avaliando o teor de nutrientes na matéria seca da parte aérea (MSPA) de plantas de jatobá na adubação completa. Próximos aos obtidos por Marques et al. (2004) para Schizolobium amazonicum com teores entre $2,7 \mathrm{~g} \mathrm{~kg}^{-1} \mathrm{e} 2,2 \mathrm{~g}$ $\mathrm{kg}^{-1} \mathrm{em}$ solução nutritiva completa e por Viégas et al. (2002) para Annona muricata com teores de 2,3 $\mathrm{g} \mathrm{kg}^{-1}$ com aplicação desse nutriente em solução nutritiva completa.

Verifica-se que todos os micros, exceto $\mathrm{Mn}$, no controle tiveram teores dentro da faixa adequada preconizada por Malavolta, Vitti e Oliveira (1997) para essências nativas (Tabela 4). Os teores foliares de Mn foram relativamente altos considerando os teores adequados preconizados por Malavolta, Vitti e Oliveira (1997) de 15 a $60 \mathrm{mg} \mathrm{kg}^{-1}$, no entanto, altas concentrações de Mn estão de acordo com o comportamento das essências florestais (REISSMANN et al., 1983).

Com relação aos elementos $\mathrm{B}, \mathrm{Cu}, \mathrm{Fe}$ e $\mathrm{Zn}$, os valores encontrados para os teores desses micronutrientes na parte aérea da lobeira na melhor combinação de AM e AO (Tabela 4) estão dentro da faixa de variação considerada adequada entre 10 a 70 para o $\mathrm{B}, 10$ a 70 para o $\mathrm{Cu}$, de 25 a 200 para o $\mathrm{Fe}$, de 5 a 60 mg kg-1 para o Zn para espécies florestais (MALAVOLTA; VITTI; OLIVEIRA, 1997). O teor adequado desses micronutrientes na parte aérea da espécie em estudo também explica o seu maior crescimento nesse tratamento, uma vez que, apesar de suas baixas concentrações dentro dos tecidos e dos órgãos das plantas, os micronutrientes têm a mesma importância dos macronutrientes para a nutrição (KIRKBY; RÖMHELD, 2007). Enquanto o $\mathrm{Cu}$ atua em diversos processos fisiológicos, o $\mathrm{Zn}$ atua nos processos metabólicos das plantas, já o B atua na dominância das gemas apicais e crescimento das plantas e o Fe que atua na constituição das enzimas (KIRKBY; RÖMHELD, 2007).

Verifica-se que os valores acumulados de nutrientes pela espécie no controle foram sempre inferiores quando comparados ao tratamento com maior crescimento (24\% AM e $76 \%$ AO) (Tabela 4), isso 
se deve, de modo geral, à menor produção de massa seca da parte aérea no controle (Figura 1). Os valores acumulados para os macronutrientes na parte aérea apresentaram a seguinte ordem de acúmulo: $\mathrm{N}>\mathrm{K}>$ $\mathrm{Ca}>\mathrm{P}>\mathrm{S}>\mathrm{Mg}$ (Tabela 5).

TABELA 5: Equações de regressão ajustadas para o acúmulo de macro e micronutrientes na parte aérea de lobeira em função da porcentagem de adubação mineral aplicada em rejeito da mineração de quartzito, acúmulo máximo de adubação mineral (AM) de nutrientes e no controle, num período de 120 dias.

TABLE 5: Regression equations fitted to the accumulation of macro and micronutrients in the shoot of lobeira according to the percentage of mineral fertilizer applied to reject from mining of quartzite, maximum accumulation (AM) of nutrients and control over a period of 120 days.

\begin{tabular}{|c|c|c|c|c|}
\hline Nutriente $^{(1)}$ & Equação de Regressão & $\mathrm{R}^{2}$ & $\mathrm{AM}^{(2)}$ & Controle $^{(3)}$ \\
\hline $\mathrm{N}$ & $\hat{\mathrm{y}}^{-1}=0,06-0,002865^{* *} \mathrm{x}+0,000054 * * \mathrm{x}^{2}$ & 0,96 & 44,75 & $6,25^{*}$ \\
\hline $\mathrm{P}$ & $\hat{y}^{-1}=0,29-0,012891 * * x+0,000234 * * x^{2}$ & 0,93 & 8,67 & $0,13^{*}$ \\
\hline $\mathrm{K}$ & $\hat{\mathrm{y}}^{-1}=0,07+0,005647 * * \mathrm{x}-0,035932 * * \mathrm{x}^{0,5}$ & 0,99 & 33,9 & $3,41^{*}$ \\
\hline $\mathrm{Ca}$ & $\hat{\mathrm{y}}^{-1}=0,19+0,005065^{* *} \mathrm{x}-0,040735^{* *} \mathrm{x}^{0,5}$ & 0,99 & 9,93 & $4,65^{*}$ \\
\hline $\mathrm{Mg}$ & $\hat{\mathrm{y}}^{-1}=1,78+0,037762 * * \mathrm{x}-0,344338^{* *} \mathrm{x}^{0,5}$ & 0,9 & 1 & $0,27 *$ \\
\hline $\mathrm{S}$ & $\hat{\mathrm{y}}^{-1}=0,23-0,019383 * * \mathrm{x}+0,000723 * * \mathrm{x}^{2}$ & 0,99 & 5,52 & $0,24 *$ \\
\hline B & $\hat{y}^{-1}=0,01-0,000469 * * x+0,000014 * * x^{2}$ & 0,98 & 146,89 & $23,50^{*}$ \\
\hline $\mathrm{Cu}$ & $\hat{\mathrm{y}}^{-1}=0,06+0,002584 * * \mathrm{x}-0,017679 * * \mathrm{x}^{0,5}$ & 0,94 & 28,24 & 9,83 \\
\hline $\mathrm{Fe}$ & $\hat{y}=61,47+4,061372 * * x-0,040180 * * x^{2}$ & 0,9 & 135,8 & $46,17^{*}$ \\
\hline $\mathrm{Mn}$ & $\hat{y}=446,09-4,789705 * * x$ & 0,88 & 331,14 & $59,55^{*}$ \\
\hline $\mathrm{Zn}$ & $\hat{y}^{-1}=0,09-0,005731^{* *} \mathrm{x}+0,000124 * * \mathrm{x}^{2}$ & 0,99 & 3,34 & $1,71 *$ \\
\hline
\end{tabular}

Em que: ** significativo a $1 \%$ pelo teste de $\mathrm{t} ;{ }^{(1)}$ Macronutrientes em mg planta ${ }^{-1}$ e micronutrientes em $\mu$ g planta ${ }^{-1} ;{ }^{(2)}$ Acúmulo máximo na aplicação de $24 \% \mathrm{AM}$ e 76 \% adubação orgânica (AO); ${ }^{(3)} *$ Diferença do controle dos demais tratamentos pelo teste $\mathrm{F}$ a $5 \%$.

Dentre os micronutrientes, na parte aérea a ordem decrescente das quantidades acumuladas ocorreu para $\mathrm{Mn}>\mathrm{B}>\mathrm{Fe}>\mathrm{Cu}>\mathrm{Zn}$ (Tabela 5). A ordem no acúmulo de macronutrientes na parte aérea da lobeira na melhor combinação de AM e AO foram similares àquela observada por Duboc et al. (1996) em folhas de mudas de jatobá submetidas na adubação completa na seguinte ordem: $\mathrm{N}>\mathrm{Ca}>\mathrm{K}>\mathrm{Mg}>\mathrm{S}>\mathrm{P}>\mathrm{Fe}$ $>\mathrm{Mn}>\mathrm{Zn}>\mathrm{Cu}$.

Não existe informação detalhada quanto ao acúmulo de nutrientes por lobeira em áreas degradadas pela de extração de quartzito. As quantidades de nutrientes alocados na biomassa vegetal devem ser consideradas, uma vez que, o conhecimento acerca do acúmulo de nutrientes pela espécie em ambiente degradado é importante, não só para o entendimento da dinâmica dos nutrientes, mas principalmente para 0 estabelecimento de práticas para a recuperação de sítios degradados (SOUZA; DAVIDE, 2001). 


\section{CONCLUSÕES}

A espécie nativa Solanum lycocarpum respondeu à aplicação da adubação orgânica e mineral com as doses recomendadas de $0,036 \mathrm{~g} \mathrm{~N}, 0,168 \mathrm{~g} \mathrm{P}_{2} \mathrm{O}_{5}$ e $0,060 \mathrm{~g}$ de $\mathrm{K}_{2} \mathrm{O}$ e 2,5 g de esterco de curral por $\mathrm{dm}^{3} \mathrm{de}$ rejeito da mineração de quartzito.

Os teores foliares de $\mathrm{N}, \mathrm{P}, \mathrm{K}, \mathrm{Ca}, \mathrm{Mg}, \mathrm{S}$ em g kg-1 e, B, Cu, Fe, Mn, e Zn em mg kg-1 na parte aérea de Solanum lycocarpum foram 21,$75 ; 4,64 ; 16,91 ; 6,78 ; 0,79 ; 2,84 ; 68,01 ; 18,69 ; 176,10 ; 262,67 ; 16,31$; respectivamente, no melhor tratamento.

$\mathrm{O}$ acúmulo de nutrientes pela espécie Solanum lycocarpum nas doses recomendadas foi na seguinte ordem na parte aérea: $\mathrm{N}>\mathrm{K}>\mathrm{Ca}>\mathrm{P}>\mathrm{S}>\mathrm{Mg}>\mathrm{Mn}>\mathrm{B}>\mathrm{Fe}>\mathrm{Cu}>\mathrm{Zn}$

\section{AGRADECIMENTOS}

Ao Conselho Nacional de Desenvolvimento Cientifico e Tecnológico pela bolsa de mestrado. A Universidade Federal dos Vales Jequitinhonha e Mucuri pela infraestrutura necessária á condução do experimento.

\section{REFERÊNCIAS}

ALVAREZ V., V. H.; RIBEIRO, A. C. Calagem. In: RIBEIRO, A. C.; GUIMARÃES, P. T. G.; ALVAREZ V., V. H.(Ed.). Recomendação para o uso de corretivos e fertilizantes em Minas Gerais. Viçosa, MG: CFSEMG, 1999. p. 43-60.

ALVES, R. J. V. et al. Circumscribing campo rupestre megadiverse Brazilian rocky montane savanas. Brazilian Journal of Biology, São Carlos, v. 74, n. 2, p. 355-362, 2014.

BEZERRA, M. W. Avaliação do processo de recuperação florestal da bacia do Rio Pipiripau. 2016. Monografia (Graduação em Engenharia Florestal) - Universidade de Brasília, Brasília, 2016.

BHATTI, J. S.; COMEFORD, N. B.; JOHNSTON, C. T. Influence of oxalate and soil organic matter on sorption and desorption of phosphate onto a Spodic horizon. Soil Science Society America Journal, Madison, v. 62, n. 4, p. 1089-1095, 1998.

CALGARO, H. F. et al. Adubação química e orgânica na recuperação da fertilidade de subsolo degradado e na micorrização do Stryphnodendron polyphyllum. Revista Brasileira Ciência do Solo, Viçosa, MG, v. 32, n. 3, p. 1337-1347, 2008.

CANTARUTTI, R. B. Pastagens. In: RIBEIRO, A. C.; GUIMARÃES, P. T. G.; ALVAREZ, V. H. (Ed.). Recomendações para o uso de corretivos e fertilizantes em Minas Gerais. Viçosa, MG: CFSEMG, 1999. p. 332-341.

CARMO, F. F. et al. Cangas: ilhas de ferro estratégicas para a conservação. Ciência Hoje, São Paulo, v. 295, p. 49-53, 2012.

CASSOL, P. C.; GIANELLO, C.; COSTA, V. E. U. Frações de fósforo em estrumes e sua eficiência como adubo fosfatado. Revista Brasileira Ciência do Solo, Viçosa, MG, v. 25, n. 3, p. 635-644, 2012.

COMISSÃO DE FERTILIDADE DO SOLO DO ESTADO DE MINAS GERAIS. Adubação orgânica. In: RIBEIRO, A. C.; GUIMARÃES, P. T. G.; ALVAREZ, V. H. (Ed.). Recomendações para o uso de corretivos e fertilizantes em Minas Gerais. Viçosa, MG: CFSEMG, 1999. p. 87-92.

CULLEN, W. R.; WHEATER, C. P.; DUNLEAVY, P. J. Establishment of species-rich vegetation on reclaimed limestone quarry faces in Derbyshire, UK. Biological Conservation, Boston, v. 84, n. 1, p. 25-33, 1998.

DANIEL, O; VITORINO, A. C. T.; ALOVISI, A. A. Aplicação de fósforo em mudas de Acacia mangiun WILL). Revista Árvore, Viçosa, MG, v. 21, n. 2, p. 163-168, 1997.

DUBOC, E. et al. Nutrição do jatobá (Hymenaea courbarilL. var. stilbocarpa (Hayne) Lee et Lang.). Cerne, Lavras, v. 2, n. 1, p. 1-12, 1996.

DUBOC, E.; GUERRINI, I. A. Concentração foliar de espécies arbóreas nativas do Cerrado sob adubação 
com nitrogênio e fósforo. In: SIMPÓSIO NACIONAL CERRADO, 2., 2008, Brasília. Anais... Planaltina: Embrapa Cerrados, 2008. (CD-ROOM).

DURÃES, M. C. O. Caracterização dos impactos ambientais da mineração na bacia hidrográfica do rio São Lamberto, Montes Claros/MG. Caderno de Ciências Agrárias, [s. 1.], v. 9, n. 1, p. 49-61, 2017. EMBRAPA. Centro Nacional de Pesquisa de Solos. Manual de métodos de análise de solo. Rio de Janeiro: CNPS, 1997. 212 p.

FALCÃO, N. P. S.; SILVA, J. R. A. Características de adsorção de fósforo em alguns solos da Amazônia Central. Acta Amazônica, Manaus, v. 34, n. 3, p. 337-342, 2004.

GONÇALVES, J. L. M. et al. Reflexos do cultivo mínimo e intensivo do solo em sua fertilidade e na nutrição das árvores. In: GONÇALVES, J. L. M.; BENEDETTI, V. (Ed.). Nutrição e fertilização florestal. Piracicaba: Instituto de Pesquisas e Estudos Florestais, 2000. p. 3-57.

GRANT, C. A. et al. A importância do fósforo no desenvolvimento inicial da planta. Informações Agronômicas, Piracicaba, n. 95, p. 1-5, 2001.

HOLL, K. D. Tropical montane forest restoration in Costa Rica: overcoming barries to dispersal and estabilishment. Restoration Ecology, Malden, v. 8, n. 2, p. 339-349, 2000.

JESUS, E. N. D. et al. Regeneração natural de espécies vegetais em jazidas revegetadas. [s. 1.: s. n.], 2016.

KANG, B. T. Changes in soil chemical proprieties and crop performance with continuos cropping on an Entisol in the humid tropics. In: MULONGOY, K.; MERCKX, R. (Ed.). Soil organic matter dynamics sustainability of tropical agriculture. New York: J. Wiley, 1991. p. 297-305.

KIRKBY, E. A.; RÖMHELD, V. Micronutrientes na fisiologia de plantas: funções absorção e mobilidade. Informações Agronômicas, Piracicaba, n. 118, p. 1-24, 2007.

MACHADO, V. J. et al. Curvas de disponibilidade de fósforo em solos com diferentes texturas após aplicação de doses crescentes de fosfato monoamônico. Bioscience Journal, Uberlândia, v. 27, n. 1, p. 70-76, 2011.

MALAVOlTA, E.; VITTI, G. C.; OLIVEIRA, S. A. Avaliação do estado nutricional das plantas: Princípios e aplicações. 2. ed. Piracicaba: POTAFOS, 1997. 319 p.

MARQUES, T. C. L. L. M. et al. Exigências nutricionais do paricá (Schizolobium amazonicum, Herb.) na fase de muda. Cerne, Lavras, v. 10, n .2, p. 167-183, 2004.

MARSCHNER, H. Mineral nutrition of higher plants. 2. ed. London: Academic Press, 1995. 889 p.

MELO, L. C. A.; SILVA, C. A. Influência de métodos de digestão e massa de amostra na recuperação de nutrientes em resíduos orgânicos. Química Nova, São Paulo, v. 31, n. 3, p. 556-561, 2008.

MORRISON, D. F. Multivariate statical methods. New York: McGraw-Hill Book, 1967. 415 p.

OLIVEIRA, F. F. Plantio de espécies nativas e uso de poleiros artificiais na restauração de uma área perturbada de cerrado sentido restrito em ambiente urbano no Distrito Federal, Brasil. 2006. 125 f. Dissertação (Mestrado em Ecologia) - Universidade de Brasília, Brasília, 2006.

OLIVEIRA JUNIOR, E. N. et al. Análise nutricional da fruta-de-lobo (Solanumlycocarpum St. Hil.) durante o amadurecimento. Ciência e Agrotecnologia, Lavras, v. 27, n. 4, p. 846-851, 2003.

PAIVA, A. V. et al. Crescimento de mudas de espécies arbóreas nativas, adubadas com diferentes doses de lodo de esgoto seco e com fertilização mineral. Scientia Forestalis, Piracicaba, v. 37, n. 84, p. 499-511, 2009.

PANDOLFO, C. M. et al. Resposta da erva-mate (Ilex paraguariensisSt. Hil.) à adubação mineral eorgânica em um latossolo vermelho aluminoférrico. Ciência Florestal, Santa Maria, v. 13, n. 2, p. 37-45, 2003.

REISSMANN, C. B. et al. Bio-elementos em folhas e hastes de erva-mate (Ilex paraguariensisSt. Hill) sobre cambissolos na região de Mandirituba-Pr. Floresta, Curitiba, v. 16, n. 2, p. 49-54, 1983.

RHEINHEIMER, D. S.; ANGHINONI, I.; CONTE, E. Fósforo da biomassa microbiana em solos sob diferentes sistemas de manejo. Revista Brasileira de Ciência do Solo, Viçosa, MG, v. 24, n. 3, p. 589-597, 2000.

RIZZINI, C. T. A flora do cerrado - analise floristica das savanas centrais. In: FERRI, M. G. (Ed.). Simpósio sobre o cerrado. São Paulo: USP; Edgaard Blücher, 1963. p. 125-177.

ROESLER, R. et al. Atividade antioxidante de frutas do cerrado. Ciência Tecnologia Alimentos, Campinas, v. 27, n. 1, p. 53-60, 2007. 
SILVA, F. C. Manual de análises químicas de solos, plantas e fertilizantes. 2. ed. Brasília: Embrapa Informações Tecnológicas, 2009. 627 p.

SILVA, K. D. A. et al. Forest restoration after bauxite mining: assessment of planted tree species. Floresta e Ambiente, Seropédica, v. 23, n. 3, p. 309-319, 2016.

SORREANO, M. C. M. et al. Deficiência de macronutrientes em mudas de Sangra d'água (Crotonuru curana, Baill.). Cerne, Lavras, v. 17, n. 3, p. 347-352, 2011.

SOUZA, C. A. M. et al. Crescimento em campo de espécies florestais em diferentes condições de adubações. Ciência Florestal, Santa Maria, v. 16, n. 3, p. 243-249, 2006.

SOUZA, J. A.; DAVIDE, A.C. Deposição de serapilheira e nutrientes em uma mata não minerada e em plantações de bracatinga (Mimosa scabrella) e de eucalipto (Eucalyptus saligna) em áreas de mineração de bauxita. Revista Cerne, Lavras, v. 7, n. 1, p. 101-113, 2001.

VARANINE, Z. et al. Low molecular weight humic substances stimulate $\mathrm{H}^{+}$-ATPase activity of plasma membrane vesicles isolated from oat (Avena sativa L.) roots. Plant and Soil, Netherlands, v. 153, n. 3, p. 61-69, 1993.

VAZ, L. M. S.; GONÇALVES, J. L. M. Uso de biossólidos em povoamento de eucalipto: efeito em atributos químicos do solo, no crescimento e na absorção de nutrientes. Revista Brasileira Ciência do Solo, Viçosa, MG, v. 26, n. 3-20, p. 747-758, 2002.

VENTURIN, N. et al. Adubação mineral da candeia (Eremanthus erythropappus (DC.) McLeish). Floresta, Curitiba, v. 35, n. 2. p. 211-219, 2005.

VIÉGAS, I. J. M. et al. Avaliação dos teores de N, P, K, Ca, Mg e S em plantas de gravioleira cultivadas em solução nutritiva com omissão de macronutrientes. Revista de Ciências Agrárias, Belém, n. 38, p. 17-28, 2002.

VIEIRA, L. S. et al. Formas de fósforo em solos do Estado do Pará - I - Latossolo Amarelo, textura média da parte Noroeste da Região Bragantina. Boletim da FCAP, Pará, v. 21, p. 65-75, 1993.

WALLAU, R. L. R. et al. Sintomas de deficiências nutricionais em mudas de mogno cultivadas em solução nutritiva. Cerne, Lavras, v. 14, n. 4, p. 304-310, 2008. 